\title{
Comparação clínica e radiológica de pulpotomia em dentes decíduos com
}

\section{formocresol, MTA e biodentine}

\author{
Clinical and radiographic comparision of primary teeth pulpotomy using formocresol, MTA and
}

biodentine

Comparación clínica y radiológica de pulpotomía de dientes primarios con formocresol, MTA y

biodentine

Recebido: 06/10/2021 | Revisado: 12/10/2021 | Aceito: 15/10/2021 | Publicado: 18/10/2021

\author{
Larissa Maria Monteiro de Albuquerque \\ ORCID: https://orcid.org/0000-0001-5138-7813 \\ Universidade Federal de Pernambuco, Brasil \\ E-mail:1malarissa1@gmail.com \\ Camila Carla de Andrade Silva \\ ORCID: https://orcid.org/0000-0001-5906-017X \\ Universidade Federal de Pernambuco, Brasil \\ E-mail: camila.andrad@hotmail.com \\ Wenys Cláudio Gomes da Silva \\ ORCID: https://orcid.org/0000-0002-9321-183X \\ Universidade Federal de Pernambuco, Brasil \\ E-mail: wenys.claudio@ufpe.br \\ Ana Cláudia da Silva Araújo \\ ORCID: https://orcid.org/0000-0002-9171-592X \\ Universidade Federal de Pernambuco, Brasil \\ E-mail: acsaodonto@gmail.com
}

\begin{abstract}
Resumo
Introdução: O processo de pulpotomia convencionalmente é feito com formocresol, porém, devido as suas características mutagênicas, novos materiais obturadores foram desenvolvidos como alternativa. Objetivo: Revisar a literatura de forma integrativa a respeito do material obturador mais adequado para pulpotomia de dentes decíduos. Metodologia: A busca ocorreu nas bases de dados Pubmed e Medline, por meio dos descritores: "child", "children", "primary teeth", "tooth, deciduous", "primary tooth", "pulpotomy", "pediatric dentistry", "endodontics", "root canal filling materials", "materials", "material", "biodentine", "mineral trioxide aggregate", "formocresol", "formocresols", "cytotoxicity", "biocompatible materials", "biocompatibility", "radiopacity", "radiographic" e "radiography, dental". Foram incluídos ensaios clínicos, estudos retrospectivos e estudos prospectivos, publicados entre 2016 e 2021, em inglês ou português. Os critérios de exclusão foram teses, dissertações, monografias, cartas ao editor, livros e revisões de literatura. Resultados: A pesquisa resultou em 9 estudos de ambas as bases de dados, com maior prevalência de ensaios clínicos. Conclusão: O material mais recomendado para realização de pulpotomias em dentes decíduos é o Biodentine e Mineral Trioxide Aggregate, devendo a escolha ser feita de acordo com o critério do profissional e caso do paciente. É preciso que seja considerado os custos e colaboração do paciente durante o tratamento.
\end{abstract}

Palavras-chave: Dente decíduo; Pulpotomia; Endodontia.

\begin{abstract}
Introduction: The pulpotomy procedure is conventionally done with formocresol, however, due to its mutagenic characteristics, new filling materials were developed as an alternative. Objective: The aim of this study was to review the literature in an integrative way regarding the most suitable filling material for pulpotomy in primary teeth. Methodology: The databases searched were Pubmed and Medline. The descriptors used for search were: "child", "children", "primary teeth", "tooth, deciduous", "primary tooth", "pulpotomy", "pediatric dentistry", "endodontics", "root canal filling materials", "materials", "material", "biodentine", "mineral trioxide aggregate", "formocresol", "formocresols", "cytotoxicity", "biocompatible materials", "biocompatibility", "radiopacity", "radiographic" and "radiography, dental". The inclusion criteria were clinical studies, retrospective studies and prospective studies published from 2016 to 2021, in English or Portuguese. The exclusion criteria were thesis, dissertations, monographs, letters to the editor, textbooks and literature reviews. Results: After the search of the articles, 9 articles from both databases were included in the literature review, with higher prevalence of clinical trials. Conclusion: The most recommended filling materials for pulpotomies in primary teeth are Biodentine and Mineral Trioxide Aggregate. The filling material must be chosen according to the criteria of the professional and the patient's case. It also needs to be considered the costs and the collaboration of the patient during the treatment.
\end{abstract}

Keywords: Primary teeth; Pulpotomy; Endodontics. 


\begin{abstract}
Resumen
Introducción: El procedimiento de pulpotomía se realiza convencionalmente con formocresol, sin embargo, debido a sus características mutagénicas, se desarrollaron nuevos materiales de obturación como alternativa. Objetivo: Realizar una revisión integradora de la literatura sobre el material de obturación más adecuado para la pulpotomía de los dientes primários. Metodología: La búsqueda se realizó en las bases de datos Pubmed y Medline, utilizando los descriptores: "child", "children", "primary teeth", "tooth, deciduous", "primary tooth", "pulpotomy", "pediatric dentistry", "endodontics", "root canal filling materials", "materials", "material", "biodentine", "mineral trioxide aggregate", "formocresol", "formocresols", "cytotoxicity", "biocompatible materials", "biocompatibility", "radiopacity", "radiographic" y "radiography, dental". Se incluyeron ensayos clínicos, estudios retrospectivos y estudios prospectivos, publicados entre 2016 y 2021, en inglés o portugués. Los criterios de exclusión fueron tesis, disertaciones, monografías, cartas al editor, libros y reseñas de literatura. Resultados: La búsqueda resultó en 9 artículos de ambas bases de datos, con una mayor prevalencia de ensayos clínicos. Conclusión: El material más recomendado para la realización de pulpotomías en dientes primarios es Biodentine y Mineral Trioxide Aggregate. La elección debe hacerse de acuerdo con el criterio del profesional y el caso del paciente. Es necesario considerar los costos y la cooperación del paciente durante el tratamiento.
\end{abstract}

Palabras clave: Diente primário; Pulpotomía; Endodoncia.

\title{
1. Introdução
}

A cárie dentária é um grande obstáculo para a saúde pública, tanto em países em desenvolvimento como nos industrializados. O tratamento da cárie pode ser realizado pela retirada do tecido infectado e cobertura da região com material restaurador. Contudo, quando há comprometimento da polpa coronária, é necessária a realização de tratamento endodôntico, como a pulpotomia (Thomas et al, 2016).

A pulpotomia é um tratamento conservador que consiste na remoção total da polpa coronária, mantendo a vitalidade e funcionalidade da polpa radicular ou de estímulos para a reparação dos dentes (Clin. Oral Investig. 2019). O procedimento é considerado bem-sucedido quando há falta de dor, sensibilidade, inchaço, radioluscência de furca e nenhum dano ao elemento dentário sucessor permanente (Gadallah et al, 2018).

O procedimento de pulpotomia é realizado tradicionalmente com o formocresol, principalmente em dentes decíduos, desde 1996, e apresenta excelente sucesso clínico de longo prazo e facilidade de manuseio. Ele apresenta, em sua formulação, concentração de formalina, em torno de 19 a 43\%. O tricresol formalina é usado como curativo de demora na câmara pulpar de dentes permanentes com necrose pulpar e lesão periapical. Este medicamento propicia a desinfecção do canal, dando condições para reparar as lesões periapicais (Juneja et al, 2017).

Apesar de viável, o formocresol é composto por formaldeído, substância que apresenta mutagenicidade e toxidade, podendo agravar mecanismos carcinogênicos, sendo, assim, o seu uso questionado na Odontologia (Alencar, 2018; Milnes, 2006; Casas, 2005; Siqueira et al, 1999; Souza et al, 1978).

Desde a sua comercialização, o MTA (Mineral Trioxide Aggregate) apresenta diversas evidências na literatura sobre a sua melhor performance e maior sucesso clínico e radiográfico do que o formocresol em pulpotomias de dentes decíduos devido a sua biocompatibilidade e bioatividade (American Academy of Pediatric Dentistry, 2020; Mestieri et al, 2015). Apesar disso, o MTA apresenta limitações como alteração na coloração do dente, longo tempo para endurecimento, dificuldade de manipulação e maior custo (Yang et al, 2021; Subay et al, 2013; Çelik et al, 2019).

O Biodentine é um cemento de silicato de cálcio que também surgiu como alternativa ao formocresol em pulpotomias de dentes decíduos devido a sua maior biocompatibilidade, tempo de preparação e menor custo (Mestieri et al, 2015). Mesmo tendo componentes similares ao MTA, o Biodentine garante estrutura menos porosa e mais densa, além de poder ser aplicado diretamente na polpa dentária (Koubi et al, 2012).

Dessa forma, o objetivo do presente estudo foi realizar uma revisão integrativa de literatura com a finalidade de comparar o sucesso clínico e radiográfico do formocresol, MTA e Biodentine como materiais obturadores em pulpotomias em dentes decíduos. 


\section{Metodologia}

Esse estudo corresponde a uma revisão integrativa de literatura, feita no ano de 2021, com o intuito de responder a seguinte pergunta condutora: entre formocresol, MTA e Biodentine, qual o melhor material obturador para realizar pulpotomia em dentes decíduos, considerando avaliação clínica e radiográfica?

Foram definidos os seguintes critérios de inclusão para selecionar os estudos: estudos publicados em português ou inglês; publicações disponíveis de estudos prospectivos, retrospectivos e ensaios clínicos, no período de janeiro de 2016 a julho de 2021 que se adequassem à pergunta condutora. Os critérios de exclusão foram teses, dissertações, monografias, cartas ao editor, livros e revisões de literatura. A coleta de dados aconteceu no mês de julho de 2021, utilizando a estratégia PRISMA para selecionar os artigos (Moher et al, 2015).

Para obter as publicações, foi realizada uma busca online nas bases de dados PubMed (National Library of Medicine) e MEDLINE (Sistema Online de Busca e Análise de Literatura Médica) via BVS (Biblioteca Virtual em Saúde). A busca foi realizada por dois autores com base na literatura selecionada, que foi analisada para definir se seria ou não incluída nesse estudo. As listas foram comparadas e, no caso de divergências, foi consultado um terceiro autor para decidir a pertinência da publicação. Após isso, foram aplicados os critérios de inclusão e exclusão.

Para a busca no PubMed, foram utilizados os descritores definidos para a pesquisa por meio da estratégia PICO disponíveis na própria plataforma como "All Fields" e os descritores disponíveis no MeSH (Medical Subject Headings). Para a população foram utilizados: "child" (All Fields), "children” (All Fields), "primary teeth" (All Fields), "tooth, deciduous" (MeSH Terms) e "primary tooth" (All Fields) combinados pelo booleano "OR"; para a intervenção foram utilizados: "pulpotomy" (MeSH Terms), "pediatric dentistry" (MeSH Terms) e "endodontics" (MeSH Terms) combinados pelo booleano "OR"; para a comparação foram utilizados: "root canal filling materials" (MeSH Terms), "materials" (All Fields), "material" (All Fields), "biodentine" (All Fields), "mineral trioxide aggregate" (All Fields), "formocresol" (All Fields) e "formocresols" (MeSH Terms) combinados pelo booleano "OR"; para o outcome foram utilizados: "cytotoxicity" (All Fields), "biocompatible materials" (MeSH Terms), "biocompatibility" (All Fields), "radiopacity" (All Fields), "radiographic" (All Fields), "radiography, dental" (MeSH Terms) combinados pelo booleano "OR". Para refinar a pesquisa, os grupos referentes à população, intervenção, comparação e outcome foram combinados por meio do operador booleano "AND", obtendo 37 artigos.

Para a busca no MEDLINE via Portal Regional da BVS, foram utilizados os descritores definidos para a pesquisa por meio da estratégia PICO disponíveis na própria plataforma como "All Fields" e os descritores disponíveis no MeSH (Medical Subject Headings). Para a população foram utilizados: "tooth, decíduos", "primary teeth", "primary tooth" combinados pelo booleano "OR"; para a intervenção foram utilizados: "pulpotomy"; para comparação foram utilizados: "root canal filling materials", "biodentine", "mineral trioxide aggregate", "formocresol" combinados pelo booleano "OR"; para o outcome foram utilizados: "cytotoxicity", "biocompatible materials", "biocompatibility", "radiopacity", "radiographic", "radiography dental" combinados pelo booleano "OR". Para refinar a pesquisa, os grupos referentes à população, intervenção, comparação e outcome foram combinados por meio do operador booleano "AND", obtendo 41 artigos.

Os estudos que atenderam aos critérios de inclusão e exclusão foram organizados para análise por um arquivo elaborado pelos autores no programa Microsoft Word contendo o seguinte: autores, título da pesquisa, ano de publicação, país, população, amostra, objetivo, métodos, resultados e conclusão do estudo.

\section{Resultados}

Por meio da estratégia de busca utilizada nesta revisão, inicialmente encontrou-se 499 artigos no PUBMED e 263 artigos na MEDLINE. A seleção resultou em 112 estudos sobre o tema, sendo eleitos 9 artigos. A Figura I demonstra o processo de 
seleção dos estudos, detalhando os critérios de elegibilidade utilizados. Todos estudos revisados estão contemplados no Quadro 1.

Figura 1: Detalhes da seleção dos estudos.
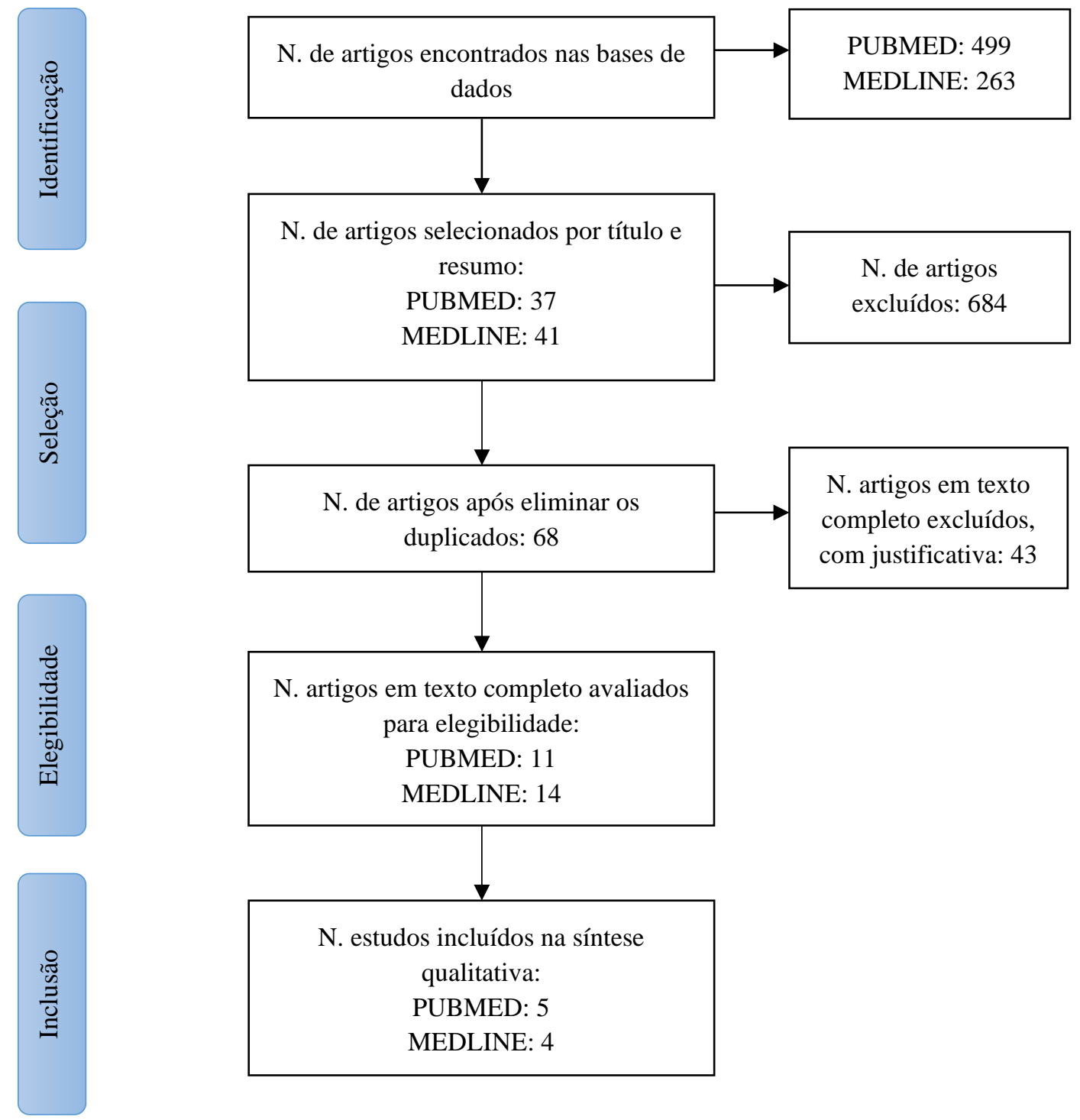

Fonte: Autores (2021). 
Quadro 1: Síntese dos artigos incluídos na pesquisa, contendo: Autor/ano, Título, Tipo de estudo, Objetivo, Métodos, Resultados e Conclusão.

\begin{tabular}{|c|c|c|c|c|c|c|}
\hline $\begin{array}{c}\text { Autor, ano, } \\
\text { país }\end{array}$ & Título & $\begin{array}{l}\text { Tipo de } \\
\text { estudo }\end{array}$ & Objetivo & Métodos & Resultados & Conclusão \\
\hline $\begin{array}{l}\text { Meligy, O. } \\
\text { A. E. S. et al } \\
\text { (2019), } \\
\text { Arábia } \\
\text { Saudita }\end{array}$ & $\begin{array}{c}\text { Biodentine TM } \\
\text { versus } \\
\text { formocresol } \\
\text { pulpotomy } \\
\text { technique in } \\
\text { primary molars: a } \\
\text { 12-month } \\
\text { randomized } \\
\text { controlled clinical } \\
\text { trial }\end{array}$ & $\begin{array}{c}\text { Ensaio } \\
\text { clínico } \\
\text { randomizad } \\
\text { o duplo cego }\end{array}$ & $\begin{array}{c}\text { Comparar o } \\
\text { sucesso clínico } \\
\text { e radiográfico } \\
\text { do Biodentine } \\
\text { com } \\
\text { formocresol. }\end{array}$ & $\begin{array}{l}112 \text { molares foram } \\
\text { separados em dois } \\
\text { grupos para } \\
\text { tratamento com } \\
\text { Biodentine e } \\
\text { formocresol, com } \\
\text { acompanhamento } \\
\text { em após } 3,6 \text { e } 12 \\
\text { meses. }\end{array}$ & $\begin{array}{c}\text { Ambos apresentaram } \\
\text { sucesso clínico de } \\
100 \% \text {. O sucesso } \\
\text { radiográfico do } \\
\text { Biodentine foi de } 100 \% \\
\text { e o do formocresol foi } \\
\text { de } 98,1 \% \text { após } 12 \\
\text { meses. }\end{array}$ & $\begin{array}{c}\text { Biodentine e } \\
\text { formocresol } \\
\text { são adequados } \\
\text { para } \\
\text { pulpotomias } \\
\text { de dentes } \\
\text { decíduos. }\end{array}$ \\
\hline $\begin{array}{l}\text { Carti, O.; } \\
\text { Oznurhan, } \\
\text { F. (2017), } \\
\text { Turquia }\end{array}$ & $\begin{array}{l}\text { Evaluation and } \\
\text { comparison of } \\
\text { mineral trioxide } \\
\text { aggregate and } \\
\text { Biodentine in } \\
\text { primary tooth } \\
\text { pulpotomy: } \\
\text { Clinical and } \\
\text { radiographic } \\
\text { study }\end{array}$ & $\begin{array}{c}\text { Ensaio } \\
\text { clínico } \\
\text { randomizad } \\
\text { o }\end{array}$ & $\begin{array}{l}\text { Comparar o } \\
\text { sucesso clínico } \\
\text { e radiográfico } \\
\text { do MTA com } \\
\text { Biodentine. }\end{array}$ & $\begin{array}{l}50 \text { molares foram } \\
\text { separados em dois } \\
\text { grupos para } \\
\text { tratamento com } \\
\text { MTA e Biodentine, } \\
\text { com } \\
\text { acompanhamento } \\
\text { após } 1,3,6 \text { e } 12 \\
\text { meses. }\end{array}$ & $\begin{array}{l}\text { Ambos apresentaram } \\
\text { sucesso clínico de } 96 \% \text {. } \\
\text { MTA teve sucesso } \\
\text { radiográfico de } 80 \% \text { e } \\
\text { Biodentine de } 60 \% \text { ao } \\
\text { final dos } 12 \text { meses. }\end{array}$ & $\begin{array}{c}\text { MTA e } \\
\text { Biodentine } \\
\text { são adequados } \\
\text { para } \\
\text { pulpotomias } \\
\text { de dentes } \\
\text { decíduos. }\end{array}$ \\
\hline $\begin{array}{l}\text { Ghoniem, } \\
\text { N. et al } \\
(2018), \\
\text { Estados } \\
\text { Unidos }\end{array}$ & $\begin{array}{l}\text { Mineral Trioxide } \\
\text { Aggregate and } \\
\text { Diluted } \\
\text { Formocresol } \\
\text { Pulpotomy: } \\
\text { Prospective and } \\
\text { Retrospective } \\
\text { Study Outcomes } \\
\end{array}$ & $\begin{array}{c}\text { Estudo } \\
\text { Biodentine e } \\
\text { retrospectiv } \\
\text { o }\end{array}$ & $\begin{array}{l}\text { Comparar o uso } \\
\text { do MTA com } \\
\text { Formocresol } \\
\text { diluído (DFC). }\end{array}$ & $\begin{array}{l}206 \text { molares foram } \\
\text { separados em dois } \\
\text { grupos para } \\
\text { tratamento com } \\
\text { MTA e DFC, com } \\
\text { acompanhamento } \\
\text { após } 48 \text { meses. }\end{array}$ & $\begin{array}{c}\text { DFC teve sucesso } \\
\text { radiográfico de } 80 \% \text {, } \\
\text { enquanto o MTA foi de } \\
90 \% \text {. }\end{array}$ & $\begin{array}{c}\text { O MTA } \\
\text { mostrou-se } \\
\text { eficiente para } \\
\text { realização de } \\
\text { pulpotomias } \\
\text { de dentes } \\
\text { decíduos. }\end{array}$ \\
\hline $\begin{array}{l}\text { Çelik, B. N. } \\
\text { et al (2019), } \\
\text { Turquia }\end{array}$ & $\begin{array}{l}\text { The evaluation of } \\
\text { MTA and } \\
\text { Biodentine as a } \\
\text { pulpotomy } \\
\text { materials for } \\
\text { carious exposures } \\
\text { in primary teeth }\end{array}$ & $\begin{array}{c}\text { Ensaio } \\
\text { clínico } \\
\text { randomizad } \\
\quad \text { o }\end{array}$ & $\begin{array}{l}\text { Comparar o } \\
\text { sucesso clínico } \\
\text { e radiográfico a } \\
\text { longo prazo do } \\
\text { Biodentine com } \\
\text { MTA. }\end{array}$ & $\begin{array}{l}44 \text { molares foram } \\
\text { separados em dois } \\
\text { grupos para } \\
\text { tratamento com } \\
\text { Biodentine e MTA, } \\
\text { com } \\
\text { acompanhamento } \\
\text { após } 3,6,12,18 \text { e } \\
24 \text { meses. }\end{array}$ & $\begin{array}{c}\text { MTA teve sucesso } \\
\text { clínico e radiográfico de } \\
100 \% \text { e o Biodentine de } \\
89,4 \% .\end{array}$ & $\begin{array}{c}\text { Biodentine é } \\
\text { mais } \\
\text { recomendado } \\
\text { para } \\
\text { pulpotomias } \\
\text { de dentes } \\
\text { decíduos pelo } \\
\text { menor tempo } \\
\text { de preparo e } \\
\text { maior } \\
\text { facilidade de } \\
\text { manipulação } \\
\end{array}$ \\
\hline $\begin{array}{l}\text { Guven, Y. } \\
\text { et al (2017), } \\
\text { Turquia }\end{array}$ & $\begin{array}{l}\text { Success Rates of } \\
\text { Pulpotomies in } \\
\text { Primary Molars } \\
\text { Using Calcium } \\
\text { Silicate-Based } \\
\text { Materials: A } \\
\text { Randomized } \\
\text { Control Trial }\end{array}$ & $\begin{array}{c}\text { Ensaio } \\
\text { clínico } \\
\text { randomizad } \\
\quad 0\end{array}$ & $\begin{array}{c}\text { Comparar } \\
\text { clinica e } \\
\text { radiográficamen } \\
\text { te o efeito de } \\
\text { materiais com } \\
\text { base de silicato } \\
\text { de cálcio com } \\
\text { sulfato férrico } \\
\text { (FS). }\end{array}$ & $\begin{array}{l}116 \text { molares foram } \\
\text { separados para } \\
\text { tratamento com } \\
\text { Biodentine, MTA-P, } \\
\text { PR-MTA e FS. }\end{array}$ & $\begin{array}{c}\text { O sucesso foi de } \\
82,75 \%, 86,2 \%, 93,1 \% \\
\text { e } 75,86 \% \\
\text { respectivamente ao final } \\
\text { de } 24 \text { meses. }\end{array}$ & $\begin{array}{l}\text { Materiais com } \\
\text { base de } \\
\text { silicato de } \\
\text { cálcio } \\
\text { mostraram-se } \\
\text { mais } \\
\text { adequados } \\
\text { para a prática } \\
\text { odontológica } \\
\text { que FS. }\end{array}$ \\
\hline $\begin{array}{l}\text { Juneja, P.; } \\
\text { Kulkarni, S. } \\
\text { (2017), } \\
\text { India }\end{array}$ & $\begin{array}{l}\text { Clinical and } \\
\text { radiographic } \\
\text { comparison of } \\
\text { Biodentine, } \\
\text { mineral trioxide } \\
\text { aggregate and } \\
\text { formocresol as } \\
\text { pulpotomy agents } \\
\text { in primary molars }\end{array}$ & $\begin{array}{c}\text { Ensaio } \\
\text { clínico } \\
\text { randomizad } \\
\text { o }\end{array}$ & $\begin{array}{c}\text { Comparar o } \\
\text { sucesso clínico } \\
\text { e radiográfico } \\
\text { do Biodentine, } \\
\text { MTA e } \\
\text { formocresol. }\end{array}$ & $\begin{array}{l}51 \text { molares decíduos } \\
\text { atribuídos } \\
\text { aleatoriamente aos } \\
\text { grupos experimental } \\
\text { ou controle. Após a } \\
\text { remoção da polpa } \\
\text { coronária e } \\
\text { hemostasia, o tecido } \\
\text { pulpar remanescente } \\
\text { foi coberto com } \\
\text { Biodentine ou } \\
\text { agregado de } \\
\end{array}$ & $\begin{array}{c}\text { Os dentes disponíveis } \\
\text { para MTA e Biodentine } \\
\text { foram clinicamente } \\
\text { bem-sucedidos. A taxa } \\
\text { de sucesso radiográfico } \\
\text { para o grupo } \\
\text { formocresol em } 18 \\
\text { meses de } \\
\text { acompanhamento foi de } \\
73,3,100 \% \text { para o MTA } \\
\text { e 86,6\% para o grupo } \\
\text { Biodentine. }\end{array}$ & $\begin{array}{l}\text { O MTA e o } \\
\text { Biodentine } \\
\text { apresentaram } \\
\text { resultados } \\
\text { mais } \\
\text { favoráveis } \\
\text { que o } \\
\text { formocresol. }\end{array}$ \\
\hline
\end{tabular}




\begin{tabular}{|c|c|c|c|c|c|c|}
\hline & & & & $\begin{array}{l}\text { trióxido mineral nos } \\
\text { grupos } \\
\text { experimentais. }\end{array}$ & & \\
\hline $\begin{array}{l}\text { Fernández, } \\
\text { C. C. et al } \\
\text { (2016), } \\
\text { Espanha }\end{array}$ & $\begin{array}{c}\text { Short-term } \\
\text { treatment } \\
\text { outcome of } \\
\text { pulpotomies in } \\
\text { primary molars } \\
\text { using mineral } \\
\text { trioxide aggregate } \\
\text { and Biodentine: a } \\
\text { randomized } \\
\text { clinical trial }\end{array}$ & $\begin{array}{c}\text { Ensaio } \\
\text { clínico } \\
\text { randomizad } \\
\mathbf{0}\end{array}$ & $\begin{array}{l}\text { Comparar o } \\
\text { sucesso clínico } \\
\text { do Biodentine } \\
\text { com MTA. }\end{array}$ & $\begin{array}{l}90 \text { molares decíduos } \\
\text { que requerem } \\
\text { pulpotomia foram } \\
\text { alocados } \\
\text { aleatoriamente para } \\
\text { o grupo MTA ou } \\
\text { Biodentine. }\end{array}$ & $\begin{array}{c}\text { A taxa de sucesso } \\
\text { clínico no Grupo MTA } \\
\text { após } 12 \text { meses foi de } \\
92 \% \text {, enquanto o Grupo } \\
\text { Biodentine obteve } 97 \% \text {. }\end{array}$ & $\begin{array}{l}\text { Biodentine } \\
\text { mostrou } \\
\text { resultados } \\
\text { clínicos } \\
\text { semelhantes } \\
\text { ao MTA com } \\
\text { taxas de } \\
\text { sucesso } \\
\text { comparáveis } \\
\text { quando usado } \\
\text { para } \\
\text { pulpotomias } \\
\text { de molares } \\
\text { decíduos. }\end{array}$ \\
\hline $\begin{array}{l}\text { Çelik, B. N.; } \\
\text { Sari, S. } \\
\text { (2016), } \\
\text { Turquia }\end{array}$ & $\begin{array}{c}\text { Carious Exposure } \\
\text { versus } \\
\text { Mechanical } \\
\text { Exposure for } \\
\text { MTA Pulpotomy } \\
\text { in Primary Teeth }\end{array}$ & $\begin{array}{l}\text { Ensaio } \\
\text { clínico }\end{array}$ & $\begin{array}{c}\text { Analisar o } \\
\text { sucesso clínico } \\
\text { e radiográfico } \\
\text { do MTA em } \\
\text { tratamentos de } \\
\text { cárie e em } \\
\text { exposição } \\
\text { pulpar } \\
\text { mecânica. }\end{array}$ & $\begin{array}{l}50 \text { dentes separados } \\
\text { em dois grupos com } \\
\text { base na exposição à } \\
\text { carie e exposição ao } \\
\text { procedimento. Foi } \\
\text { realizada avaliação } \\
\text { após } 3,6,12 \text { e } 18 .\end{array}$ & $\begin{array}{l}\text { O sucesso clínico foi de } \\
\text { 100\% em ambos os } \\
\text { grupos. }\end{array}$ & $\begin{array}{l}\text { A taxa de } \\
100 \% \text { de } \\
\text { sucesso } \\
\text { indicando que } \\
\text { o MTA pode } \\
\text { ser usado em } \\
\text { casos de } \\
\text { contaminação } \\
\text { bacteriana e } \\
\text { resposta } \\
\text { inflamatória. }\end{array}$ \\
\hline $\begin{array}{l}\text { Junqueira, } \\
\text { M. A. et al } \\
\text { (2018), } \\
\text { Brasil }\end{array}$ & $\begin{array}{c}\text { Clinical, } \\
\text { Radiographic and } \\
\text { Histological } \\
\text { Evaluation of } \\
\text { Primary Teeth } \\
\text { Pulpotomy Using } \\
\text { MTA And Ferric } \\
\text { Sulfate }\end{array}$ & $\begin{array}{l}\text { Ensaio } \\
\text { clínico }\end{array}$ & $\begin{array}{c}\text { Avaliar } \\
\text { resultados } \\
\text { clínicos, } \\
\text { radiográficos e } \\
\text { histológicos do } \\
\text { MTA com o } \\
\text { sulfato férrico } \\
\text { (SF). }\end{array}$ & $\begin{array}{l}31 \text { molares decíduos } \\
\text { alocados } \\
\text { aleatoriamente nos } \\
\text { grupos MTA ou SF. } \\
\text { As avaliações } \\
\text { clínicas e } \\
\text { radiográficas, com } \\
\text { acompanhamentoap } \\
\text { ós } 3,6,12 \text { e } 18 \\
\text { meses. }\end{array}$ & $\begin{array}{c}\text { Ambos os grupos } \\
\text { exibiram } 100 \% \text { de } \\
\text { sucesso radiográfico aos } \\
3,6 \text { e } 12 \text { meses. } \\
\text { Histologicamente, os } \\
\text { dentes tratados } \\
\text { apresentaram vitalidade } \\
\text { pulpar e ausência de } \\
\text { infiltrado inflamatório } \\
\text { no tecido conjuntivo. }\end{array}$ & $\begin{array}{c}\text { Ambos os } \\
\text { materiais são } \\
\text { adequados } \\
\text { para } \\
\text { pulpotomia } \\
\text { em dentes } \\
\text { decíduos. }\end{array}$ \\
\hline
\end{tabular}

Fonte: Autores (2021).

\section{Discussão}

A cárie dentária é uma doença crônica que acomete crianças no mundo todo. A sua evolução pode acarretar em comprometimento pulpar e, caso se torne irreversível, é necessária a realização de procedimento endodôntico para preservar a integridade do elemento dentário e evitar exodontia. A pulpotomia é um tratamento conservador realizado em dentes com vitalidade pulpar, em que a infecção bacteriana é retirada e a cavidade pulpar é preenchida com material obturador (Clin. Oral Investig., 2019; Thomas, 2016).

Desde a sua introdução na indústria, o formocresol foi considerado o padrão ouro para o procedimento de pulpotomia graças as suas propriedades bacteriostáticas e sucesso clínico. Porém devido à atividade mutagênica proveniente do aldeído, outros materiais mais biocompatíveis são utilizados, como MTA e Biodentine, ambos com base de silicato de cálcio (Siqueira et al, 1999; Goyal, 2014; Carti, 2017).

O formocresol apresenta sucesso clínico variando de 73,3\% a $100 \%$ e clínico de $73,3 \%$ a $98,1 \%$, o que indica a sua viabilidade como material obturador em pulpotomias (Meligy et al, 2019; Ghoniem et al, 2018; Juneja et al, 2017). Essa variação se dá pelo tamanho da amostra e, principalmente, pelo tempo de acompanhamento dos estudos, visto que, quanto maior ele for, maior será a disparidade entre os resultados encontrados.

No estudo de Ghoniem et al (2018), foi comparado a utilização do formocresol diluído (DFC) com G-MTA no período de 42 meses e constatado em um dos locais de realização do experimento que dentes tratados com DFC tiveram 5,1 vezes mais 
chances de apresentar falha do que os dentes tratados com o outro material. Entretanto, é preciso considerar que o estudo apresentou como limitação a taxa de retorno dos pacientes, que ao final do estudo chegou apenas a $6 \%$, devido à exfoliação natural dos dentes e dificuldade de transporte.

O MTA, desenvolvido como alternativa ao tratamento com formocresol, apresenta bons resultados clínicos e radiográficos, de $86,2 \%$ a $100 \%$ e de $80 \%$ a 100\%, respectivamente (Carti et al, 2017; Ghoniem et al, 2018; Çelik et al, 2019; Guven et al, 2017; Junqueira et al, 2018; Fernández et al, 2016; Juneja et al, 2017). O estudo de Çelik et al (2016) sugeriu que o material, além de biocompatível, é adequado para casos de contaminação bacteriana e com alta resposta inflamatória. Além disso, tem maior capacidade seladora, alta radiopacidade e pH de 11-12 (Carti et al, 2017).

Para Junqueira et al (2018), o uso do MTA na odontopediatria é limitado pelo seu alto custo, longo tempo de preparo e dificuldade de manipulação, considerando o tempo de permanência do dente na cavidade oral da criança. No entanto, todos os dentes tratados com o material no estudo apresentaram sucesso clínico ao final dos 12 meses de acompanhamento, além de ter sido observado vitalidade pulpar e ausência de infiltrado inflamatório no tecido conjuntivo.

Devido ao alto custo, foi desenvolvido o MTA-Plus, que possui componentes similares ao produto original e com partículas mais finas, para oferecer menor custo, menor tempo de preparo e aplicação mais fácil. Foi obtido resultado satisfatório, apesar de menos eficiente (Guven et al, 2017).

Como alternativa para o uso do MTA, foi desenvolvido o Biodentine, o qual apresenta a vantagem de ser fácil de manipular e ter alta viscosidade (Carti et al, 2017). Isso é corroborado pelo estudo de Juneja et al (2017), que sugere a boa adaptação marginal do Biodentine e resistência à compressão adequada para ser utilizado como material obturador. Além disso, facilita o tratamento na Odontopediatria porque o procedimento tem menor tempo de duração e pode ser usado como substituto permanente da dentina sob a coroa dentária em uma única sessão (Cuadros-Fernández et al, 2016).

Ao comparar o MTA com o Biodentine, Carti et al (2017) relatou que não houve diferença na avaliação clínica após 12 meses, porém na radiológica, o MTA mostrou-se superior, com sucesso de $80 \%$, enquanto o Biodentine teve $68 \%$. Esses resultados são condizentes com os de Çelik et al (2019), em que o sucesso clínico e radiográfico do MTA foi de 100\%, enquanto o de Biodentine foi de $89,4 \%$ ao final dos 24 meses.

Após a análise dos estudos disponíveis na literatura, foi observado a viabilidade do MTA e do Biodentine como materiais obturadores. O MTA apresenta maior taxa de sucesso em relação ao Biodentine, que, por sua vez, tem o benefício de ser mais barato e fácil de manipular. O formocresol continua sendo uma alternativa devido a sua popularização no meio odontológico e custo acessível.

\section{Conclusão}

O método mais recomendado para a realização de pulpotomia em dentes decíduos, de acordo com os resultados desse estudo, é o uso de Biodentine e MTA, uma vez que esses materiais possuem alta taxa de sucesso no tratamento de pulpotomias em dentes decíduos. A escolha quanto ao material utilizado deve ser feita pelo profissional após a análise do caso do paciente. O Biodentine se destaca pelo menor custo, menor duração do procedimento e resultado clínico e radiográfico satisfatório.

Como possibilidade para novas pesquisas em relação à escolha de materiais obturadores para pulpotomias em dentes decíduos, sugerimos estudos clínicos de longo prazo com amostras maiores e que avaliem o sucesso clínico e radiográfico desses materiais.

\section{Referências}

Alencar, A. V., Santos, V. E., Jr., Calixto, M. S., Santos, N., Heimer, M. V., \& Rosenblatt, A. (2018). Evaluation of the genotoxic effects of formocresol application in vital pulp therapy of primary teeth: a clinical study and meta-analysis. Clinical Oral Investigations, 22(7), $2553-2558$. 
American Academy of Pediatric Dentistry (2020). Pulp therapy for primary and immature permanent teeth. The Reference Manual of Pediatric Dentistry. American Academy of Pediatric Dentistry, 384-92.

Carti, O. \& Oznurhan, F. (2017). Evaluation and comparison of mineral trioxide aggregate and biodentine in primary tooth pulpotomy: Clinical and radiographic study. Nigerian Journal of Clinical Practice, 20(12), 1604-1609.

Casas, J. M., Kenny, D. J., Judd, P. L. \& Johnston, D. H. (2005). Do We Still Need Formocresol in Pediatric Dentistry? Journal of the Canadian Dental Association, 71(10), 749-751.

Çelik, B. N., Mutluay, M. S., Arikan, V. \& Sari, Ş. (2019). The evaluation of MTA and Biodentine as a pulpotomy materials for carious exposures in primary teeth. Clinical Oral Investigations, 23(2), 661-666.

Çelik, B. N. \& Sari, S (2016). Carious Exposure versus Mechanical Exposure for MTA Pulpotomy in Primary Teeth. BioMed Research International, 2016(3), $1-6$.

Cuadros-Fernández, C., Rodríguez, A. I. L., Sáez-Martínez, S., García-Binimelis, J., About, I. \& Mercadé, M. (2016). Short-term treatment outcome of pulpotomies in primary molars using mineral trioxide aggregate and Biodentine: a randomized clinical trial. Clinical Oral Investigation, 20(7), 639-45.

Dimitraki, D., Papageorgiou, S. N. \& Kotsanos, N. (2019) Direct pulp capping versus pulpotomy with MTA for carious primary molars: a randomised clinical trial. European Archives of Paediatric Dentistry, 20(5), 431-440.

Gadallah, L., Hamdy, M., Bardissy, A. E. \& Yazeed, M. A. M. (2018). Pulpotomy versus pulpectomy in the treatment of vital pulp exposure in primary incisors. A systematic review and meta-analysis. F1000Res, 26(7), 1-21.

Ghoniem, N. \& Vaidyanathan, V. et al (2018). Mineral Trioxide Aggregate and Diluted Formocresol Pulpotomy: Prospective and Retrospective Study Outcomes. The Journal of the Michigan Dental Association, 100(4), 40-65.

Goyal, S., Abuwala, T., Joshi, K., Mehta, J., Indushekar, K. R. \& Hallikerimath, S. (2014). The Clinical, Radiographic and Histological evaluation of three different concentrations of Formocresol as a pulpotomy agent. Journal of international oral health, 6(2), 118-125.

Guven, Y., Aksakal, S. D., Üstün, N., \& Unsal, G (2017). Success Rates of Pulpotomies in Primary Molars Using Calcium Silicate-Based Materials: A Randomized Control Trial. BioMed Research International, 2017(2), 1-7.

Juneja, P. \& Kulkarni, S. (2017). Clinical and radiographic comparison of biodentine, mineral trioxide aggregate and formocresol as pulpotomy agents in primary molars. European Archives of Paediatric Dentistry, 18(4), 271-278.

Junqueira, M. A., Cunha, N. N. O., Caixeta, F. F., Marques, N. C. T., Oliveira, T. M., Moretti, A. B. D. S., Cosme-Silva, L. \& Sakai, V. T. (2018). Clinical, Radiographic and Histological Evaluation of Primary Teeth Pulpotomy Using MTA And Ferric Sulfate. Brazilian Dental Journal, $29(2), 159-165$.

Koubi, S., Elmerini, H., Koubi, G., Tassery, H. \& Camps, J. (2012). Quantitative Evaluation by Glucose Diffusion of Microleakage in Aged Calcium SilicateBased Open-Sandwich Restorations. International Journal of Dentistry, 2012(2), 1-6.

Meligy, O. A. E. S., Alamoudi, N. M., Allazzam, S. M., \& El-Housseiny; A. A. M. (2019). BiodentineTM versus formocresol pulpotomy technique in primary molars: a 12-month randomized controlled clinical trial. BMC Oral Health, 19(1), 1-8.

Mestieri, L. B. et al (2015). Biocompatibility and bioactivity of calcium silicate-based endodontic sealers in human dental pulp cells. Journal of Applied Oral Science [online], 23(5), 467-471.

Milnes, A. R. (2006). Persuasive Evidence that Formocresol Use in Pediatric Dentistry Is Safe. Journal of the Canadian Dental Association, 72(3), 247-248f.

Moher, D., Shamseer, L., Clarke, M., Ghersi, D., Liberati, A., Petticrew, M., Shekelle, P., \& Stewart, L. A. (2015). Preferred Reporting Items for Systematic Review and Meta-Analysis Protocols (PRISMA-P) 2015 statement. Syst Rev, 4(1):1.

Subay, R. K., Ilhan, B.\& Ulukapi, H. (2013) Mineral trioxide aggregate as a pulpotomy agent in immature teeth: Long-term case report. European Journal of Dentistry, 7, 133-138.

Yang, W. C., Tsai, L. Y., Hsu, Y. H., Teng, N. C., Yang, J. C., \& Hsieh, S. C. (2021). Tooth discoloration and the effects of internal bleaching on the novel endodontic filling material SavDen® MTA. Journal of the Formosan Medical Association, 120(1), 476-482 